PACIFIC JOURNAL OF MATHEMATICS

Vol. 178, No. 2, 1997

\title{
EQUIVALENCE OF ANALYTIC AND SOBOLEV POINCARÉ INEQUALITIES FOR PLANAR DOMAINS
}

\author{
Alexander Stanoyevitch and David A. Stegenga \\ For a finitely connected planar domain $\Omega$ it is shown that \\ the analytic-Poincaré inequality

$$
\left\|f(z)-f\left(z_{0}\right)\right\|_{L^{p}(\Omega)} \leq K_{p}^{a}(\Omega)\left\|f^{\prime}(z)\right\|_{L^{p}(\Omega)}
$$ \\ holds uniformly for all holomorphic functions $f$ on $\Omega\left(z_{0} \in \Omega\right.$ \\ fixed, $K_{p}^{a}(\Omega)$ an absolute constant) if and only if the Sobolev- \\ Poincaré inequality $\|u(z)\|_{L^{p}(\Omega)} \leq K_{p}(\Omega)\|\nabla u(z)\|_{L^{p}(\Omega)}$ holds for \\ an absolute constant $K_{p}(\Omega)$ and for all $u \in \mathcal{C}^{1}(\Omega)$ whose integral \\ over $\Omega$ is zero. This paper extends a result of Hamilton (1986) \\ who established this equivalence when $1<p<\infty$.
}

\section{Introduction.}

Let $\Omega \subseteq \mathbb{R}^{2}$ be a domain, i.e., an open connected set, of finite area $|\Omega|$. For an exponent $p, 1 \leq p \leq \infty$ we say that $\Omega$ is an analytic p-Poincaré domain provided that there exists a constant $K_{p}^{a}(\Omega)$ such that the analytic p-Poincaré inequality

$$
\left\|f(z)-f\left(z_{0}\right)\right\|_{L^{p}(\Omega)} \leq K_{p}^{a}(\Omega)\left\|f^{\prime}(z)\right\|_{L^{p}(\Omega)}
$$

holds for each holomorphic function $f \in H(\Omega)$. Here $z_{0}$ is any fixed "base point" in $\Omega$. It is easy to check that the validity of $(1)$ is independent of the choice of $z_{0}$, although the best constant in (1) will depend on $z_{0}$. The domain $\Omega$ is called a Sobolev p-Poincaré domain if there exists an absolute constant $K_{p}(\Omega)$ such that the Sobolev p-Poincaré inequality

$$
\left\|u(z)-u_{\Omega}\right\|_{L^{p}(\Omega)} \leq K_{p}(\Omega)\|\nabla u(z)\|_{L^{p}(\Omega)}
$$

holds for all smooth functions $u \in \mathcal{C}^{1}(\Omega) \cap L^{p}(\Omega)$. We are letting $u_{\Omega}$ denote the average value of $u$ over $\Omega$, i.e.,

$$
u_{\Omega}=\frac{1}{|\Omega|} \int_{\Omega} u(z) d A(z) .
$$

The Poincaré inequalities are useful for an assortment of applications so it is useful to know when Poincaré inequalities hold on a given domain $\Omega$. We 
shall see (in Section 2) that the analytic $p$-Poincaré inequality (1) is equivalent to the validity of (2) with the functions $u$ being restricted to be in $H(\Omega) \cap L^{p}(\Omega)$. Thus, the analytic Poincaré inequalities can be viewed as weaker versions of the corresponding Sobolev-Poincaré inequalities where the "test functions" are assumed to be analytic. With this formulation it is clear that if $\Omega$ is a Sobolev $p$-Poincaré domain then it is also an analytic $p$-Poincaré domain. Hamilton [Ham] showed that the reverse implication holds provided $1<p<\infty$. Actually his result was stated with the assumptions $p=2$ and $\Omega$ is simply connected but all that his proof needs is $1<p<\infty$ (no topological assumptions on $\Omega$ are required). The main tool in his proof is the boundedness of the Hilbert and Cauchy transforms on $L^{p}$, and since these transforms are badly unbounded on $L^{1}$ and $L^{\infty}$ his proof yields no information on the borderline cases $p=1$ and $p=\infty$. Making use of more explicit constructions, we shall prove the following:

Main Theorem. If $p=1$ or $p=\infty$ and if $\Omega \subseteq \mathbb{R}^{2}$ is a finitely connected analytic p-Poincaré domain, then $\Omega$ is a Sobolev p-Poincaré domain.

In proving this theorem, the point that will allow us to pass from simply connected to finitely connected domains is that the finitely many components of $\partial \Omega$ cannot work together to thwart the Poincaré inequality (either the analytic or Sobolev version) if one of them could not do the job separately. For domains of infinite connectivity, the interrelationships of the individual boundary components becomes pertinent and the present approach of constructing appropriate analytic test functions does not apply. The result should, however, still be true.

Question. Are the analytic and Sobolev p-Poincaré inequalities equivalent for arbitrary planar domains of finite area?

The Sobolev $p$-Poincaré inequality (2) makes sense for $n$-dimensional domains $\Omega \subseteq \mathbb{R}^{n}$ of finite volume. These Poincaré inequalities have numerous applications, for example, it is well known that the validity of (2) for $p=2$ is tantamount to the solvability of the Neumann problem with arbitrary data. For details on this see $\S 4.10$ in $[\mathrm{Maz}]$ and $\S 4$ in [Sta]. Thus it is often required to determine if a given domain $\Omega$ supports a $p$-Poincaré inequality. We have shown that in 2-dimensions (at least for finitely connected domains) the class of test functions needed to verify (2) can be reduced from the class of smooth functions $\mathcal{C}^{1}(\Omega) \cap L^{p}(\Omega)$ to the much smaller class of analytic functions $H(\Omega) \cap L^{p}(\Omega)$. It would be interesting and useful to know if an analogous reduction is valid in higher dimensions.

Question. For a domain $\Omega \subseteq \mathbb{R}^{n}(n>2)$ of finite volume, is it sufficient to test the $p$-Poincaré inequalities (2) on the class of harmonic functions in 
$L^{p}(\Omega)$ ? How about the class of quasiconformal functions in $L^{p}(\Omega)$ ?

\section{The Case $p=1$.}

The first order of business will be to observe that the analytic 1-Poincaré inequality is equivalent to the validity of (2) with the test functions $u$ being restricted to lie in $H(\Omega) \cap L^{1}(\Omega)$. Indeed for such a test function $u$, and a given base point $z_{0}$, the mean value property gives that

$$
u\left(z_{0}\right)=u_{B}
$$

where $B \subset \Omega$ is any disk centered at $z_{0}$. The above equivalence is thus a consequence of the following

Lemma. If $u \in L^{1}(\Omega)$ and $A \subset \Omega$ is a measurable set of positive area then

$$
\begin{aligned}
\left(1+\frac{|\Omega|}{|A|}\right)^{-1} \iint_{\Omega}\left|u(x, y)-u_{A}\right| d x d y & \leq \iint_{\Omega}\left|u(x, y)-u_{\Omega}\right| d x d y \\
& \leq 2 \iint_{\Omega}\left|u(x, y)-u_{A}\right| d x d y
\end{aligned}
$$

Proof. Using the triangle inequality, we have

$$
\begin{aligned}
\iint_{\Omega}\left|u-u_{\Omega}\right| & \leq \iint_{\Omega}\left(\left|u-u_{A}\right|+\left|u_{A}-u_{\Omega}\right|\right) \\
& =\iint_{\Omega}\left|u-u_{A}\right|+|\Omega|\left|u_{A}-u_{\Omega}\right| .
\end{aligned}
$$

Now,

$$
\begin{aligned}
\left|u_{A}-u_{\Omega}\right| & =\left|u_{A}-\frac{1}{|\Omega|} \iint_{\Omega} u(x, y) d x d y\right| \\
& =\left|\frac{1}{|\Omega|} \iint_{\Omega}\left(u(x, y)-u_{A}\right)\right| \\
& \leq \frac{1}{|\Omega|} \iint_{\Omega}\left|u-u_{A}\right| .
\end{aligned}
$$

Combining these two estimates produces the second inequality of the Lemma. The first inequality is obtained in a similar fashion. 
Remark. A similar argument would yield the corresponding equivalence for $p$-Poincaré domains for all $p \geq 1$.

Our approach to proving the Main Theorem will be to assume $\Omega$ is not a Sobolev 1-Poincaré domain and to construct explicit analytic functions which would force the constant $K_{1}^{a}(\Omega)$ in the analytic 1-Poincaré inequality (1) to be arbitrarily large. To accomplish this construction we shall need some sort of geometric consequence of $\Omega$ failing to be a Sololev 1-Poincaré domain. This will be provided by the following geometric characterization of simply connected Sobolev 1-Poincaré domains which appeared as part of Theorem A in [StSt].

Theorem. If $\Omega \subseteq \mathbb{R}^{2}$ is a simply connected domain of finite area, then $\Omega$ is a Sobolev 1-Poincaré domain if and only if $L<\infty$ where

$$
L=\sup \left\{\frac{\left|\Omega_{\sigma}\right|}{\ell(\sigma)}: \sigma \text { a segmental crosscut of } \Omega\right\} .
$$

Here $\Omega_{\sigma}$ is a subdomain of $\Omega \backslash \sigma$ of minimal area and $\ell(\sigma)$ denotes the length of the segment $\sigma$. Moreover, the best (i.e., smallest) constant $K_{1}(\Omega)$ that works in (2) is comparable to $L$.

The construction will be separated into three stages. First we deal with $\Omega$ being a Jordan domain with a polygonal boundary. Next we use this special case together with a normal families argument to establish the result for arbitrary simply connected domains. The proof will be completed by using the simply connected case to prove the general result for finitely connected domains. We now fix a base point $z_{0} \in \Omega$ and for convenience we assume that $|\Omega|=1$.

Step $1 . \quad \Omega$ is a polygonal Jordan domain.

Let $\sigma$ be a "short" segmental crosscut of $\Omega$ with $\ell(\sigma)$ much less than $\delta_{\Omega}\left(z_{0}\right)$, where $=\operatorname{dist}\left\{z_{0}, \partial \Omega\right\}$ and much less than $\left|\Omega_{\sigma}\right|$. By the previously mentioned theorem it will suffice to construct a function $f \in H(\Omega) \cap L^{1}(\Omega)$ which satisfies

$$
\iint_{\Omega}\left|f(z)-f\left(z_{0}\right)\right| d A(z) \gtrsim\left|\Omega_{\sigma}\right|
$$

and

$$
\iint_{\Omega}\left|f^{\prime}(z)\right| d A(z)=O(\ell(\sigma))
$$

The symbol " $\gtrsim$ " means greater than or equal to modulo an absolute constant. We also use the notation $a \approx b$ to denote $a \lesssim b \lesssim a$. 
By an isometric change of variables, we can write

$$
\sigma=[-\eta, \eta], \quad \text { with } \eta>0 .
$$

Note that by our assumption on $\sigma$, $\operatorname{dist}\left\{\sigma, z_{0}\right\}$ is much greater than $\eta$.

We let $\Omega_{\sigma}\left(z_{0}\right)$ denote the component of $\Omega \backslash \sigma$ which contains $z_{0}$, and set $\Gamma=\partial \Omega_{\sigma}\left(z_{0}\right) \backslash \sigma$. Since $\Gamma$ is itself a (polygonal) Jordan arc, its complement $G=\widehat{\mathbb{C}} \backslash \Gamma$ is simply connected and it follows (from [Rud, Theorem 13.11] along with an inversion) that there exists a holomorphic square root of the nonvanishing analytic function $z^{2}-\eta^{2}$. We denote this function by $\sqrt{z^{2}-\eta^{2}}$.

Consider the function

$$
f(z)=\frac{z}{\sqrt{z^{2}-\eta^{2}}} \in H(G) .
$$

Clearly

$$
\operatorname{dist}(f(z),\{ \pm 1\})<\frac{1}{2} \quad \text { for }|z|>M \eta
$$

where $M>0$ is sufficiently large. An economical choice for $M$ could easily be obtained but we do not require such precision. All that is important for our purposes is to note that a choice for $M$ can be made which is independent of $\eta$.

From the continuity of $f$ on $\Omega$, it follows that a single sign in (7) will persist in each component of $\Omega \backslash \operatorname{Ball}(0, M \eta)$. For definiteness we assume that $f\left(z_{0}\right)$ is close to 1 in the sense of (7) (if not, replace $\sqrt{z^{2}-\eta^{2}}$ with $\left.-\sqrt{z^{2}-\eta^{2}}\right)$.

We let $\left\langle D_{j}\right\rangle_{j=1}^{k}$ denote the (finitely many) components of $\Omega \backslash \overline{\operatorname{Ball}(0, M \eta)}$. Note that each $D_{j}$ is itself a Jordan domain whose boundary $\partial D_{j}$ consists of a subset of $\partial \operatorname{Ball}(0, M \eta)$ together with a subset of either $\Gamma$ or $\partial \Omega \backslash \Gamma$ (but not both!). We partition the $D_{j}$ 's into two categories: $\left\langle A_{i}\right\rangle_{j=1}^{k_{A}},\left\langle B_{j}\right\rangle_{j=1}^{k_{B}}$ where each $A_{i}$ satisfies $\partial A_{i} \cap \Gamma \neq \emptyset$ and each $B_{j}$ satisfies $\partial B_{j} \cap \Gamma=\emptyset$. For each $j \in\left\{1, \ldots, k_{B}\right\}$ we let $\gamma_{j}$ denote a path in $\mathbb{C} \backslash \overline{\operatorname{Ball}(0, M \eta)}$ which joins $z_{0}$ to $B_{j}$ and which crosses $\Gamma$ transversally a finite number, $n_{j}$, of times.

We next use the fact that $\Gamma$ is the natural boundary of our function $\sqrt{z^{2}-\eta^{2}}$ and furthermore, each time $z$ crosses $\Gamma$ (at a nonendpoint, i.e., at a point $\neq \pm \eta$ ) the sign of $\sqrt{z^{2}-\eta^{2}}$ reverses. Indeed, suppose that this fails for some point $w_{0} \in \Gamma$. It follows from a routine analytic continuation argument that our function $\sqrt{z^{2}-\eta^{2}}$ would have an analytic extension to all of $\mathbb{C} \backslash\{ \pm \eta\}$, and hence (as these singularities are removable) to all of $\mathbb{C}$. Thus, $z^{2}-\eta^{2}$ is the square of some entire function. This is impossible since this function has simple zeros. 
It follows that each time $\gamma_{j}$ crosses $\Gamma$, the sign in (7) changes. Also since an even number of crossings $\left(n_{j}\right)$ would result in landing in an $A_{i}$, it follows that $n_{j}$ is odd for all $j$, and consequently

$$
\left|f(z)-f\left(z_{0}\right)\right| \geq 1 \quad\left(z \in \cup B_{j}\right) .
$$

We claim that

$$
\left|\cup B_{j}\right| \gtrsim\left|\Omega_{\sigma}\right| \text {. }
$$

There are two cases depending on which of the two components of $\Omega \backslash \sigma$ has the larger area. If $z_{0} \in \Omega_{\sigma}$ then $\left|\cup B_{j}\right|$ is large, at least $1 / 4$ for small $\eta$, and so (9) is clear. On the other hand, if $z_{0} \notin \Omega_{\sigma}$, then

$$
\left|\Omega_{\sigma}\right| \leq\left|\cup B_{j}\right|+|\operatorname{Ball}(0, M \eta)|
$$

and again (9) follows since our assumption is that $\eta$ is small compared to the area of $\Omega_{\sigma}$.

Using (8) and (9) we conclude

$$
\begin{aligned}
\iint_{\Omega}\left|f(z)-f\left(z_{0}\right)\right| d A(z) & \geq \iint_{B_{j}}\left|f(z)-f\left(z_{0}\right)\right| d A(z) \\
& \geq\left|\cup B_{j}\right| \\
& \gtrsim\left|\Omega_{\sigma}\right|
\end{aligned}
$$

which establishes (4).

Differentiating (6) yields

$$
\left|f^{\prime}(z)\right|=\frac{\eta^{2}}{|z-\eta|^{\frac{3}{2}}|z+\eta|^{\frac{3}{2}}} .
$$

To estimate the integral, we partition the domain $\Omega$ into the following four subdomains:

$$
\begin{aligned}
& \Omega_{1}=\{z \in \Omega:|z-\eta|<\eta\} \\
& \Omega_{2}=\{z \in \Omega:|z+\eta|<\eta\} \\
& \Omega_{3}=\{z \in \Omega:|z|<3 \eta\} \backslash\left\{\Omega_{1} \cup \Omega_{2}\right\} \\
& \Omega_{4}=\{z \in \Omega:|z| \geq 3 \eta\} .
\end{aligned}
$$

For $z \in \Omega_{1},|z+\eta| \approx \eta$ so by (10),

$$
\iint_{\Omega_{1}}\left|f^{\prime}(z)\right| d A(z) \approx \sqrt{\eta} \int_{0}^{2 \pi} \int_{0}^{\eta} \frac{r}{r^{\frac{3}{2}}} d z d \theta \approx \eta .
$$


By the same token we get

$$
\iint_{\Omega_{2}}\left|f^{\prime}(z)\right| d A(z) \approx \eta .
$$

For $z \in \Omega_{3},|z \pm \eta| \approx \eta$ so by $(10),\left|f^{\prime}(z)\right| \approx \frac{1}{\eta}$ and since $\left|\Omega_{3}\right| \approx \eta^{2}$ it follows that

$$
\iint_{\Omega_{3}}\left|f^{\prime}(z)\right| d A(z) \approx \eta .
$$

Finally, for $z \in \Omega_{4}$, we find $|z \pm \eta| \approx|z|$ so that $\left|f^{\prime}(z)\right| \approx \frac{\eta^{2}}{|z|^{3}}$ and hence

$$
\begin{aligned}
\iint_{\Omega_{4}}\left|f^{\prime}(z)\right| d A(z) & \lesssim \eta^{2} \int_{0}^{2 \pi} \int_{3 \eta}^{\infty} \frac{r}{r^{3}} d r d \theta \\
& \approx \eta^{2} \frac{1}{\eta}=\eta
\end{aligned}
$$

as well, and (5) is established.

Since $\ell(\sigma) \approx \eta$, we have shown that

$$
\frac{\left|\Omega_{\sigma}\right|}{\ell(\sigma)} \lesssim \frac{\iint_{\Omega}\left|f-f\left(z_{0}\right)\right| d A(z)}{\iint_{\Omega}\left|f^{\prime}(z)\right| d A(z)} \leq K_{1}^{a}(\Omega)
$$

provided that $\ell(\sigma)$ is small compared to $\delta_{\Omega}\left(z_{0}\right)$ and $\left|\Omega_{\sigma}\right|$. Hence the supremum $L$ of (3) has been shown to be finite if $\Omega$ is an analytic 1-Poincaré domain. Step 1 is complete.

Step $2 . \quad \Omega$ is a simply connected domain.

The approach will be the same as in Step 1: We let $\sigma$ be a short segmental crosscut of $\Omega$ and wish to construct a function $f \in H(\Omega) \cap L^{1}(\Omega)$ which satisfies (4) and (5).

Claim. There exists a sequence $\left\langle\Omega_{n}\right\rangle_{n=1}^{\infty}$ of polygonal Jordan domains inside $\Omega$ such that

(i) $\left|\Omega_{\sigma} \backslash \Omega_{n}\right| \longrightarrow 0, \Omega_{n} \subset \subset \Omega_{n+1}$ and

(ii) $\sigma_{n} \stackrel{D E F}{\equiv} \Omega_{n} \cap \sigma$ is a segmental crosscut of $\Omega_{n}$ with $\ell\left(\sigma_{n}\right) \longrightarrow \ell(\sigma)$.

The proof of the Claim is a straightforward construction and we omit the details.

For each $n$ we may assume $z_{0} \in \Omega_{n}$, and we let $\Omega_{n}\left(z_{0}\right)$ denote the component of $\Omega_{n} \backslash \sigma_{n}$ which contains $z_{0}$. We write

$$
\sigma_{n}=\left[a_{n}, b_{n}\right], \quad \sigma=[a, b],
$$


so that $a_{n} \searrow a, b_{n} \nearrow b$ and let

$$
f_{n}(z)=\frac{z}{\sqrt{\left(z-a_{n}\right)\left(z-b_{n}\right)}}
$$

correspond to the pair $\left\langle\Omega_{n}, z_{0}\right\rangle$ in the construction of Step 1. Note that $\left|f_{n}\left(z_{0}\right)-1\right|<\frac{1}{2}$. Now for each fixed $n_{0}$, the family $\left\langle f_{n}\right\rangle_{n>n_{0}}$ is uniformly bounded on $\overline{\Omega_{n}}$ and is hence a normal family. Thus (by Theorem 15, p. 224 in $[\mathbf{A h l}])$ there exists $f \in H(\Omega)$ such that

$$
f_{n} \longrightarrow f
$$

uniformly on compact subsets of $\Omega$. From (12) and since $a_{n} \longrightarrow a, b_{n} \longrightarrow b$ we conclude

$$
f(z)=\frac{z}{\sqrt{(z-a)(z-b)}}
$$

and hence the estimate (5) follows exactly as in the proof of Step 1. As for (4) we first note on $\Omega_{n_{0}},\left|f_{n}\right| \lesssim|f|$ for $n>n_{0}$. Hence, given $\epsilon>0$, we can find $n_{0}$ such that

$$
\int_{\Omega_{\sigma} \backslash \Omega_{n_{0}}}\left|f_{n}(z)-f_{n}\left(z_{0}\right)\right| d A(z)<\epsilon \quad\left(\text { all } n \geq n_{0}\right) .
$$

Next choose $n_{1}>n_{0}$ such that for all $n>n_{1}$

$$
\left|f(z)-f_{n}(z)\right|<\epsilon \text { on } \Omega_{n_{0}} .
$$

We may now conclude that

$$
\begin{aligned}
& \int_{\Omega}\left|f(z)-f\left(z_{0}\right)\right| d A(z) \\
& \gtrsim \int_{\Omega_{n_{0}} \backslash \operatorname{Ball}\left(\frac{a+b}{2}, M \eta\right)}\left|f(z)-f\left(z_{0}\right)\right| d A(z) \\
& \geq \int_{\Omega_{n_{0}} \backslash \operatorname{Ball}\left(\frac{a+b}{2}, M \eta\right)}\left|f_{n}(z)-f_{n}\left(z_{0}\right)\right| d A(z)-2 \epsilon \\
& \geq \int_{\Omega_{n} \backslash \operatorname{Ball}\left(\frac{a+b}{2}, M \eta\right)}\left|f_{n}(z)-f_{n}\left(z_{0}\right)\right| d A(z)-3 \epsilon \\
& \gtrsim\left|\Omega_{\sigma_{n}}\right|-3 \epsilon .
\end{aligned}
$$

Since this holds for all $n>n_{0}$, and $\epsilon>0$ we obtain

$$
\int_{\Omega}\left|f(z)-f\left(z_{0}\right)\right| d A(z) \gtrsim\left|\Omega_{\sigma}\right|,
$$


as desired.

Step $3 . \quad \Omega$ is a finitely connected domain.

Write

$$
\partial \Omega=\Gamma_{0} \cup \bigcup_{i=1}^{k} \Gamma_{i}
$$

where $\Gamma_{0}$ is the outer boundary component and each $\Gamma_{i}(i \geq 1)$ is an inner boundary component. Pick points $z_{i}$ in $\operatorname{Int}\left(\Gamma_{i}\right)(i \geq 1)$ - the interior of $\Gamma_{i}$ and let $\Gamma_{i}^{\star}$ denote the image of $\Gamma_{i}$ under the analytic inversion $z \mapsto 1 /\left(z-z_{i}\right)$. Put

$$
\Omega_{i}=\operatorname{Int}\left(\Gamma_{i}^{\star}\right) \quad(i \geq 1)
$$

and

$$
\Omega_{0}=\operatorname{Int}\left(\Gamma_{0}\right)
$$

Let us show that $\Omega_{0}$ is a 1-Poincaré domain whenever $\Omega$ is a 1-Poincaré domain. Start by constructing a simply connected domain $D \subset \Omega_{0}$ with a smooth boundary lying in $\Omega$, i.e., the holes of $\Omega$ are contained in $D$. Since $D$ has a smooth boundary, it is well known to be a 1-Poincaré domain. By the lemma, we may take averages over any disk $A \subset \Omega \backslash \Omega_{0}$ in place of $u_{\Omega}$. Given a smooth test function on $\Omega_{0}$, then since $\Omega$ and $D$ are both 1-Poincaré domains we have that

$$
\int_{\Omega_{0}}\left|u-u_{A}\right| \leq \int_{\Omega}\left|u-u_{A}\right|+\int_{D}\left|u-u_{A}\right| \lesssim \int_{\Omega_{0}}|\nabla u|
$$

and hence $\Omega_{0}$ is also a 1-Poincaré domain.

Since the above inversion mappings are all bilipschitz homeomorphisms on $\Omega$, it follows in a similar manner that the (analytic or Sobolev) Poincaré inequality holds on $\Omega$ if and only if it holds on each $\Omega_{i}(0 \leq i \leq k)$.

Hence, if $\Omega$ failed to be a Sobolev 1-Poincaré domain then so would some $\Omega_{i}$ and the construction in Step 2 would furnish analytic functions on $\Omega_{i}$ which would pull back to analytic functions on $\Omega$ (by the inversion $z \mapsto$ $1 /\left(z-z_{i}\right)$ if $i \geq 1$, and by simple restriction if $\left.i=0\right)$ which would violate the analytic Poincaré inequality (1) for any given constant $K_{1}^{a}(\Omega)$.

\section{The Case $p=\infty$.}

We first consider the reduction of the Main Result to the case of simply connected domains. The argument in the previous section works equally 
well for the case $p=\infty$ once we establish that any bounded domain with a smooth boundary is an $\infty$-Poincaré domain. One method for proving this is to apply the following proposition.

Proposition. Let $\Omega$ be any domain, $z_{0} \in \Omega$ and denote the inner distance in $\Omega$ by

$$
d_{\Omega}\left(z_{0}, z\right)=\inf \left\{\ell(\gamma): \gamma \text { curve in } \Omega \text { from } z_{0} \text { to } z\right\}
$$

If $D_{\Omega}\left(z_{0}\right)=\sup _{z \in \Omega} d_{\Omega}\left(z_{0}, z\right)<\infty$ then $\Omega$ is an $\infty$-Poincaré domain and

$$
K_{\infty}(\Omega) \leq 2 D_{\Omega}\left(z_{0}\right)
$$

Proof. For $u \in \mathcal{C}^{1}(\Omega)$ and $z \in \Omega$ we have (by Calculus) that

$$
\left|u(z)-u\left(z_{0}\right)\right| \leq \int_{\gamma}|\nabla u| d s \leq\|\nabla u\|_{L^{\infty}(\Omega)} \cdot \ell(\gamma)
$$

for any curve $\gamma$ in $\Omega$ which connects $z_{0}$ to $z$. Thus,

$$
\left|u(z)-u\left(z_{0}\right)\right| \leq D_{\Omega}\left(z_{0}\right)\|\nabla u\|_{L^{\infty}(\Omega)}
$$

which yields the result since

$$
\left|u(z)-u_{\Omega}\right| \leq\left|u(z)-u\left(z_{0}\right)\right|+\frac{1}{|\Omega|} \int_{\Omega}\left|u-u\left(z_{0}\right)\right| \leq 2 D_{\Omega}\left(z_{0}\right)\|\nabla u\|_{L^{\infty}(\Omega)} .
$$

Theorem. Let $\Omega$ be a simply connected domain with finite area and $z_{0} \in \Omega$. Then,

$$
K_{\infty}^{a}(\Omega) \approx K_{\infty}(\Omega) \approx D_{\Omega}\left(z_{0}\right)
$$

In order to prove this theorem we first observe that by the proposition and a calculation similar to one in $\S 2$ we have that

$$
K_{\infty}^{a}(\Omega) \lesssim K_{\infty}(\Omega) \lesssim D_{\Omega}\left(z_{0}\right)
$$

and hence we must prove that $D_{\Omega}\left(z_{0}\right) \lesssim K_{\infty}^{a}(\Omega)$. This requires constructing an analytic function and this is done in the next section. 


\section{The Construction of an analytic test function.}

Fix a simply connected domain $\Omega$, with nonempty boundary and let $\gamma$ be a hyperbolic geodesic in $\Omega$, i.e., $\gamma$ is the image of a diameter of the unit disk under a Riemann mapping function. Suppose further that $\left\{w_{n}\right\}_{n=-\infty}^{\infty}$ is a sequence of points on $\gamma$ satisfying

$$
\rho_{\Omega}\left(w_{n}, w_{m}\right)=n-m \quad-\infty<m<n<\infty,
$$

where $\rho_{\Omega}$ denote the hyperbolic distance in $\Omega$, see $[$ Ahl2, §1.1]. Recall that $\delta_{\Omega}(w)$ denotes the Euclidean distance to $\partial \Omega$.

Lemma. There are apriori constants $c_{1}>0, c_{2}>0,0<\alpha<1$ and an analytic function $f$ on $\Omega$ satisfying

(i) If $a \in \gamma$ precedes $w_{-1}$ and $b \in \gamma$ follows $w_{1}$, then

$$
\Re\left(\int_{\gamma(a, b)} f d w\right) \geq c_{1} \ell\left(\gamma\left(w_{-1}, w_{1}\right)\right) .
$$

(ii) Let $w \in \Omega$ and let $\tau$ be the hyperbolic geodesic through $w$ which is orthogonal to $\gamma$. If $\tau$ intersects $\gamma$ between $w_{n}$ and $w_{n+1}$, then

$$
|f(w)| \leq c_{2} \alpha^{|n|} .
$$

Proof. Let $\varphi: \Omega \rightarrow \Delta$ be the Riemann mapping function which maps $\Omega$ to the unit disk $\Delta$ and maps $\gamma$ into the imaginary axis. This mapping is uniquely determined by requiring that $\varphi\left(w_{0}\right)=0$ and $\Im \varphi\left(w_{1}\right)>0$. Let $\psi$ be the inverse mapping.

Choose $|\lambda|=1$ so that $i \lambda \psi^{\prime}(0)=\left|\psi^{\prime}(0)\right|$. Now it follows from the Koebe distortion theorem, see [Pom, $\S 1.3]$, that

$$
\Re\left(i \lambda \psi^{\prime}(i t)\right) \geq \frac{1}{2}\left|\psi^{\prime}(0)\right|
$$

for $|t|<\eta$, where $\eta>0$ is some apriori constant. Another consequence of the distortion theorem is that

$$
\left(1-t^{2}\right)^{3}\left|\psi^{\prime}(i t)\right| \leq 16\left|\psi^{\prime}(0)\right| \quad(|t|<1)
$$

Now fix an integer $p \geq 3$, which will be chosen later, and define the analytic function on $\Omega$ by

$$
f(w)=\lambda\left(\frac{1+\varphi(w)^{2}}{2}\right)^{p} \quad(w \in \Omega)
$$


To prove (i) let $\varphi(a)=t_{a} i$ and $\varphi(b)=t_{b} i$. By conformal invariance of the hyperbolic metric we know that there is an apriori positive constant, which we may as well assume is $\eta$, such that $\left|t_{a}\right|>\eta$ and $\left|t_{b}\right|>\eta$. Combining these estimates with the above ones from the distortion theorem yield that

$$
\begin{aligned}
2^{p} \Re \int_{\gamma(a, b)} f d w & =\Re \int_{t_{a}}^{t_{b}} \lambda\left(1+\varphi(\psi(i t))^{2}\right)^{p} i \psi^{\prime}(i t) d t \\
& =\int_{t_{a}}^{t_{b}}\left(1-t^{2}\right)^{p} \Re\left(i \lambda \psi^{\prime}(i t)\right) d t \\
& \geq\left[\int_{0}^{\eta}\left(1-t^{2}\right)^{p} d t-32 \int_{\eta}^{1}\left(1-t^{2}\right)^{p-3} d t\right]\left|\psi^{\prime}(0)\right| .
\end{aligned}
$$

For $p$ sufficiently large (depending on $\eta$ ) the quantity in brackets is positive. Fix such a $p$ and set $c_{1}$ equal to this quantiy divided by $2^{p}$. One more application of the distortion theorems yields that $\left|\psi^{\prime}(0)\right|$ is comparable to $\ell\left(\gamma\left(w_{0}, w_{1}\right)\right)$. Thus, (i) follows.

To prove (ii), fix $w \in \Omega$ and let $\tau$ be the geodesic in the hypothesis of (ii). For concreteness, assume that $\tau$ intersects $\gamma$ at the point $w^{\prime}$ and that $\rho_{\Omega}\left(w_{0}, w_{n}\right) \leq \rho_{\Omega}\left(w_{0}, w^{\prime}\right)<\rho_{\Omega}\left(w_{0}, w_{n+1}\right)$. Notice that this implies that $\Im \varphi\left(w^{\prime}\right)>0$. Since $\varphi(\tau)$ is a geodesic in $\Delta$ which is orthogonal to the imaginary axis it is elementary that $|i-\varphi(w)| \approx\left|i-\varphi\left(w^{\prime}\right)\right|$ and hence

$$
\begin{aligned}
|f(w)| & =\left|\frac{1+\varphi(w)^{2}}{2}\right|^{p} \leq|i-\varphi(w)|^{p} \\
& \lesssim\left|i-\varphi\left(w_{n}\right)\right|^{p} \lesssim\left(\frac{1-\left|\varphi\left(w_{n}\right)\right|}{1+\left|\varphi\left(w_{n}\right)\right|}\right)^{p} \\
& =\exp \left(-p \rho_{\Delta}\left(0, \varphi\left(w_{n}\right)\right)\right)=\exp \left(-p \rho_{\Omega}\left(w_{0}, w_{n}\right)\right)=e^{-n p} .
\end{aligned}
$$

Hence $c_{2}, \alpha$ can be chosen (depending only on $p$ ) so (ii) holds. This completes the proof.

Theorem. $D_{\Omega}\left(w_{0}\right) \lesssim K_{\infty}^{a}(\Omega)$.

Proof. Suppose that $0<D<D_{\Omega}\left(w_{0}\right)$. Then there must be a hyperbolic geodesic $\gamma$ with $\ell(\gamma)>D$. Fix such a $\gamma$ and let $\left\{w_{n}\right\}_{n=-\infty}^{\infty}$ be a sequence of points on $\gamma$ satisfying the conditions in the lemma. Removing $w_{0}$ from $\gamma$ we split $\gamma$ into two parts $\gamma_{+}$and $\gamma_{-}$based on the indices for the $w_{n}$ 's. We will assume that $\ell\left(\gamma_{+}\right) \geq D / 2$.

For each integer $n$ let $f_{n}$ be the analytic function on $\Omega$ constructed in the lemma with the point $w_{n}$ playing the role of $w_{0}$ in the lemma. Next, let

$$
g_{n}(w)=\sum_{j=1}^{n} \int_{w_{0}}^{w} f_{j} d w \quad(w \in \Omega, n=1,2, \ldots)
$$


then since $\Omega$ is simply connected the $\left\{g_{n}\right\}$ are all analytic on $\Omega$.

By part (ii) of the lemma we have $\left\|g_{n}^{\prime}\right\|_{\infty}<2 c_{2} /(1-\alpha)=c_{3}$ for all $n$. On the other hand, part (i) yields that

$$
\begin{aligned}
\Re g_{n}\left(w_{n+1}\right) & =\sum_{j=1}^{n} \Re \int_{\gamma\left(w_{0}, w_{n+1}\right)} f_{j} d w \\
& \geq \sum_{j=1}^{n} c_{1} \ell\left(\gamma\left(w_{j-1}, w_{j}\right)\right) \geq c_{1} \ell\left(\gamma\left(w_{0}, w_{n+1}\right)\right) .
\end{aligned}
$$

The analytic Poincaré inequality now implies that

$$
\left|g_{n}\left(w_{n+1}\right)\right|=\left|g_{n}\left(w_{n+1}\right)-g\left(w_{0}\right)\right| \leq K_{\infty}^{a}\left(w_{0}\right)\left\|g_{n}^{\prime}\right\|_{\infty}
$$

and combining this with the above inequality gives

$$
D \leq 2 \ell\left(\gamma_{+}\right)=2 \lim _{n \rightarrow \infty} \ell\left(\gamma\left(w_{0}, w_{n}\right)\right) \leq \frac{2 c_{3}}{c_{1}} K_{\infty}^{a}\left(w_{0}\right)
$$

which proves the theorem.

\section{References}

[Ahl] L.V. Ahlfors, Complex Analysis, Second Edition, McGraw-Hill, New York, 1966.

[Ahl2] Conformal Invariants: Topics in Geometric Function Theory, McGrawHill, New York, 1973.

[Ham] D.H. Hamilton, On the Poincaré inequality, Complex Variables Theory Appl., 5 (1986), 265-270.

[Maz] V.G. Maz'ya, Sobolev Spaces, Springer-Verlag, Berlin Heidelberg, 1985.

[Pom] Ch. Pommerenke, Boundary Behavior of Conformal Maps, Springer-Verlag, Berlin, 1992.

[Rud] W. Rudin, Real and Complex Analysis, Second Edition, McGraw-Hill, New York, 1974.

[Sta] A. Stanoyevitch, Products of Poincaré domains, Proc. Amer. Math. Soc., 117 (1993), 79-87.

[StSt] A. Stanoyevitch and D.A. Stegenga, The geometry of Poincaré disks, Complex Variables Theory Appl., 24 (1994), 249-266.

Received August 1, 1995.

UNIVERSity OF HAWAII AT MANOA

HoNOLULU, HI 96822-2273

E-mail address: stegenga@math.hawaii.edu 